\title{
NEW FEATURES OF THE INCL4 MODEL FOR SPALLATION REACTIONS
}

\author{
J. CUGNON ${ }^{*}, 1$, A. BOUDARD ${ }^{2}$, S. LERAY ${ }^{2}$, and D. MANCUSI ${ }^{1}$ \\ ${ }^{1}$ University of Liège, allée du 6 août 17, bât. B5, B-4000 Liège 1, Belgium \\ ${ }^{2}$ Irfu/SPhN, CEA-Sacaly, F-91191 Gif-sur-Yvette, Cedex, France \\ ${ }^{*}$ Corresponding author. E-mail : cugnon@plasma.theo.phys.ulg.ac.be \\ Received \\ Accepted for Publication
}

The standard version (INCL4.2) of the Liège Intra-nuclear Cascade model for spallation reactions has been shown to reproduce reasonably well a large set of experimental data, when coupled to the standard ABLA model. However, some systematic deficiencies were surviving. In order to reduce the latter, an improved model (INCL4.5) has been worked out. The improvements of the model are described here for the first time. They involve in particular the production of composite particles in the cascade and the behaviour of the model at low incident energy. Typical results are shown and discussed.

KEYWORDS : Spallation reactions, Intra-nuclear Cascade model, particle energy spectra, residue cross sections

\section{INTRODUCTION}

It is more and more accepted that the nucleon-induced spallation reactions proceed through a two stage process. The first stage is dominated by binary collisions which eject a few fast particles leaving at the end of the stage a more or less thermalized remnant. The second stage corresponds to the further de-excitation of this remnant. The standard tools to describe these stages is the IntraNuclear Cascade (INC) model for the first one and the evaporation-fission model for the second one. There are however still pending questions concerning the appropriateness of these standard tools. Some of them, concerning de-excitation, are discussed in another presentation to this conference [1]. A large effort has been done during the last decade aiming at improving the theoretical tools. In particular, as a result of the European HINDAS project [2], the Liège INCL model, denoted INCL4.2 [3], and the Karl-Heinz Schmidt ABLA fissionevaporation model [4] have emerged and it was shown that their combination is quite successful to describe the bulk of experimental data in the incident energy range spanning from $200 \mathrm{MeV}$ to $3 \mathrm{GeV}$. Yet, these models are not perfect and, for both of them, systematic shortcomings have been identified. In the last four years, within the EU EUROTRANS collaboration, both models have been improved to cope with those deficiencies. It is the purpose of this presentation to report on the improvements of the INCL model and to present, for the first time, the new version, denominated INCL4.5. A preliminary account can be found in Ref. [5] and a detailed account will be published soon.

\section{DESCRIPTION OF THE MODEL}

The standard INCL4.2 model is described in Ref. [2]. It is sufficient to recall here that INCL4.2 is a basically parameter-free time-like cascade model following the fate of all particles which undergo binary collisions, dictated by a minimum distance of approach criterion and subject to probabilistic Pauli blocking factors, and reflection or transmission at the nuclear surface. Pion production and absorption are treated through the $\Delta$ degrees of freedom. A unique feature of INCL is the self-consistent determination of the stopping time. The main deficiencies of the predictive power of INCL4.2 are: (i) based on individual degrees of freedom, it cannot accommodate cluster production, (ii) it somehow underestimates the production yield of residues close to the target (iii) pion production is somehow overestimated for heavy targets, (iv) the predictions at less than $200 \mathrm{MeV}$ are not satisfactory.

We shortly describe the new features contained in the new version INCL4.5:

-Inclusion of known phenomenology. Energy and 
isospin-dependent potentials are introduced for nucleons as well as isospin-dependent potentials for pions. They are taken from the optical-model phenomenology. Coulomb ddeflection is introduced for incoming and outgoing charged particles.

-Light charged particle production. This is now possible owing to a dynamical coalescence procedure. A nucleon crossing the nuclear surface can drag along other nucleons which are sufficiently close by in phase space to form a light cluster.

-Improvement of the Pauli blocking. Collisions between particles below the Fermi level, even if they are participants, are forbidden. A strict Pauli blocking is applied to the first collision.

-Treatment of soft collisions. In INCL4.2, collisions with low c.m. energy, that are expected to contribute to the mean field rather than to affect the energy-momentum flow, are avoided (except for the first collision). The critical c.m. energy has been reduced to $1910 \mathrm{MeV}$.

-Special 'local E' procedure. It is applied, for the first collision, if the latter occurs in the nuclear surface. It aims at compensating for the momentum content of the nuclear surface, which, in INCL4.2, is overestimated, compared to phenomenological models for the density in phase space. This procedure nevertheless satisfies all conservation laws.

- "Fuzzy" Fermi surface. In order to comply with arguments in favour of this feature, participants which fall in energy below the Fermi level plus a value, taken to be $18 \mathrm{MeV}$, are given back the status of spectators (they cannot escape unless they are hit by a participant).

\section{RESULTS}

\subsection{Observables directly linked to the cascade}

We give in Fig. 1 results for the total reaction cross section. This quantity is solely determined by the cascade. One will notice a considerable improvement of the predictions of INCL at low incident energy. This improvement is due to the careful treatment of the first collision at low energy.

Other quantities directly linked to cascade are the high energy parts (above evaporation, say above $20 \mathrm{MeV}$ ) of the $n, p$ and $l c p$ spectra. Details cannot be given here, for lack of space. An example of the capabilities of INCL4.5 is given in Fig. 2. It shows that this model describes reasonably well the production of alpha particles of 100 $\mathrm{MeV}$ energy or more.

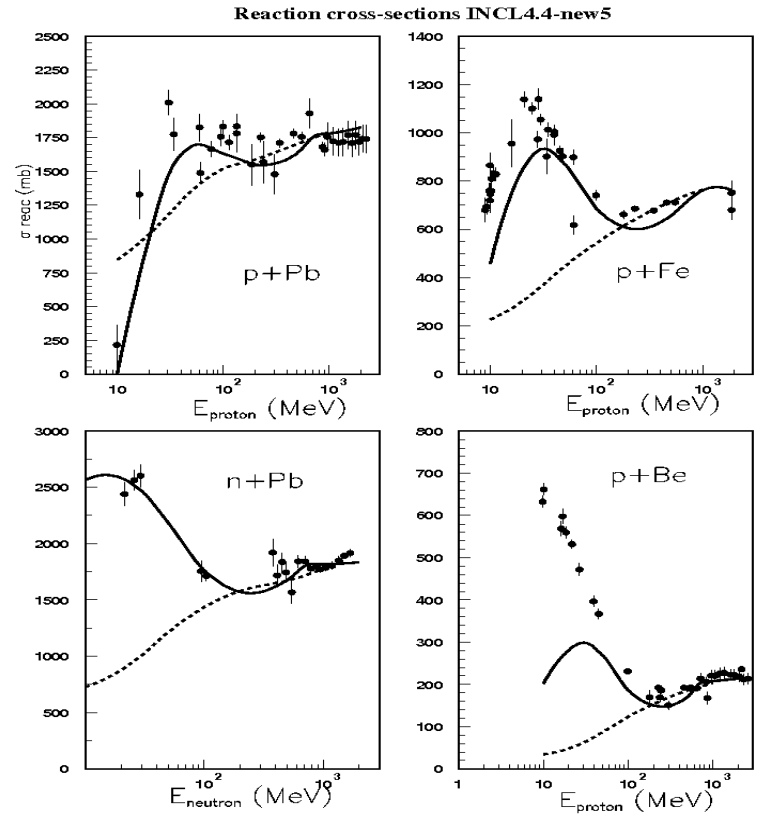

Fig. 1. Total reaction cross sections for various systems. Comparison between the predictions of INCL4.2 (dotted curves), those of INCL4.5 (full curves) and experimental data [6].

An intercomparison between several cascade+evaporation models for spallation reactions has been organized by the IAEA, benchmarking them on a vast amount of data for extended ranges in target mass and incident energy [7] . An evaluation meeting, held recently in Saclay, has demonstrated the high predictive power of INCL4.5 for this kind of observables. Yet, there are still small discrepancies remaining. For instance, proton and, to a smaller extent, neutron spectra are slightly depleted just above the evaporation peak. They are somehow "eaten" by the coalescence.

\subsection{Observables determined by cascade and de-excitation}

Among these observables are the evaporation spectra, which of course are mainly determined by the deexcitation models, but are also influenced by the properties of the remnants left by the cascade models. In the following, we will restrict to the results obtained by INCL4.5 when coupled with the de-excitation code ABL07[9], the new version of ABLA, which has been improved in the frame of the EUROTRANS collaboration also. In general, neutron and proton evaporation spectra are well reproduced by the INCL4.5-ABLA07 combination. The agreement is less good for $l c p$ spectra, especially at low incident energy. This seems to arise, partly, from a lack of coherence between the Coulomb barrier heights used in the two stages. 
model (see Ref. [3]).

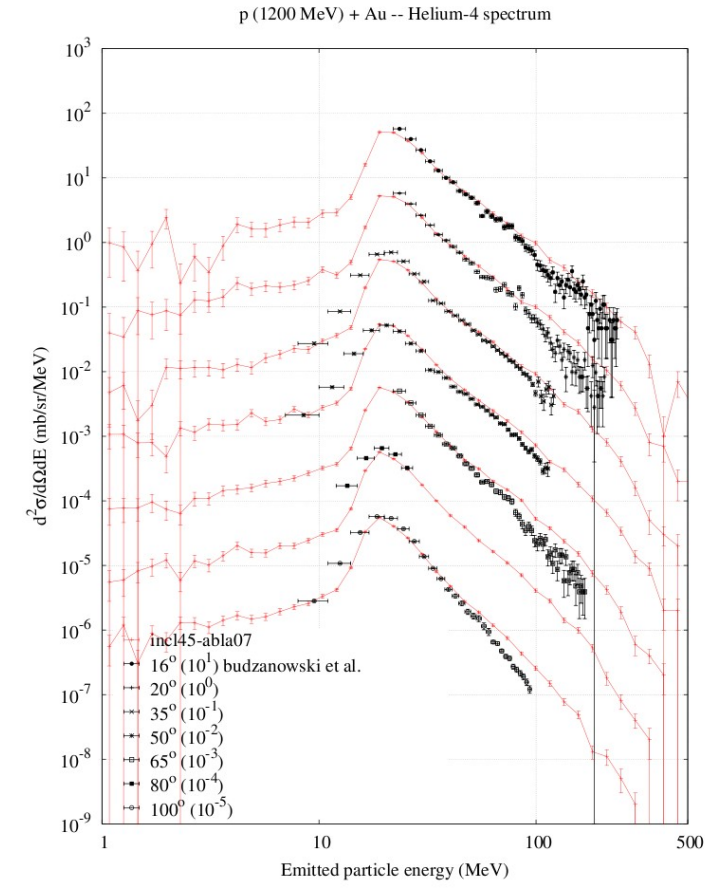

Fig. 2. Double differential cross sections for the production of alpha particles in $\mathrm{p}(1.2 \mathrm{GeV})+$ Au reactions. Comparison between the predictions of INCL4.5 coupled to ABLA07 (full curves) and experimental data [8].

Other observables of this kind are the residue yields and properties. Let us start with the residue mass spectrum. Predictions are compared with experimental data for the $\mathrm{p}(1 \mathrm{GeV})+{ }^{208} \mathrm{~Pb}$ in Fig.3. With the previous INCL4.2+ABLA version (see Ref. [3]), the low mass part of the evaporation residue peak was largely underestimated. The improvement is partly due to inclusion of $l c p$ emission, which generates remnants of higher excitation energy and lower mass and partly to the introduction of IMF emission in ABLA07, which helps to increase mass loss in de-excitation. On the other hand, the fission yield is overestimated, by $\sim 20 \%$, besides a slight underestimate of the width of the peak. This may be explained by the fact that, in this reaction, the conditions are such that the fission yield is rather rapidly varying with the fissility parameter and with excitation energy. Very likely, the fission model in ABLA07 is not constrained enough in this region.

Note, however, that the residue production yield is slightly underestimated for residues close to the target. This seems to be a persistent deficiency of the INCL4

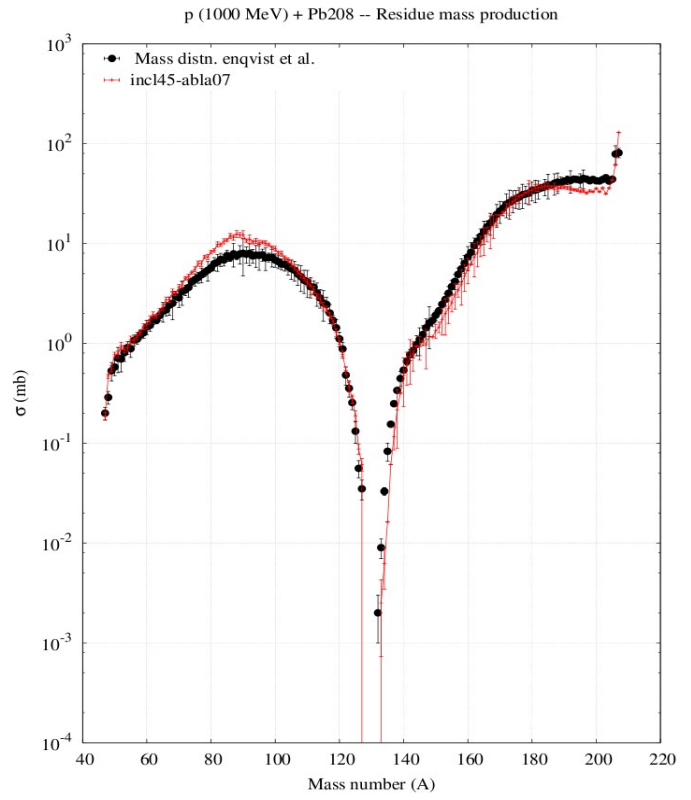

208

Fig. 3. Residue mass spectrum in $\mathrm{p}(1 \mathrm{GeV})+\mathrm{Pb}$ reactions. Comparison between the predictions of INCL4.5 coupled to ABLA07 (full curve) and experimental data [10].

Another example is provided by Fig. 4, which shows the same comparison for the case of p-induced reactions on 238

U. The agreement with the experimental data is spectacular. Of course, the merit can be attributed to ABLA07, since the data are largely dominated by fission. However, the shape and yield of the evaporation residue peak are determined by INCL4.2, which generates a correct distribution of $\mathrm{A}, \mathrm{Z}$ and excitation energy of the remnants which resist to fission. On the other hand, the shape of the fission peak is entirely due to ABLA07. It should be mentioned that the agreement has been obtained after refinements of shell effects. Results for nonfissioning systems (not shown here) have also been worked out, namely $\mathrm{p}(0.5 \mathrm{GeV})+{ }^{208} \mathrm{~Pb}$ and $\mathrm{p}(1 \mathrm{GeV})+{ }^{56}$ Fe. In the former case, the agreement is excellent, while in the latter case, a good agreement seems to require a small component of multifragmentation. The implications of the last feature is discussed in Ref. [1]. 
Residue production data have been analyzed in more detail, for instance by looking at the so-called isotopic distributions, namely the residue mass distributions for given elements. If, in general, a good agreement between model predictions and data is achieved, systematics deviations arise for the $\mathrm{p}(1 \mathrm{GeV})+{ }^{208} \mathrm{~Pb}$ case. For most of the elements produced as evaporation residues, the predicted distributions are shifted toward the neutron-rich side by 1-2 mass units. This seems to point to a wrong balance between neutron and proton evaporation, in favour of the last one. On the other hand, residues with the same charge as the target are depleted in the theoretical predictions. In addition, residues with a one or two units of charge less and with the maximum mass are overestimated. These residues are produced through (p, $\mathrm{xp}+\mathrm{yn}$ ), with $\mathrm{x}$ and $\mathrm{y}$ equal to 1 or 2 , i.e. in events with one or two NN collisions, mainly. This deficiency, which can be attributed to the cascade model only, is paradoxical as these events are those which are expected to be well described by cascade models.

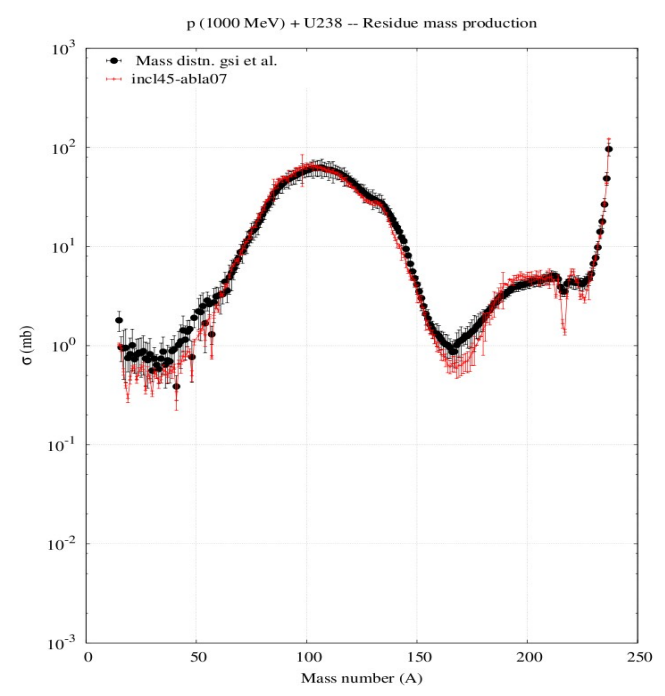

Fig. 4. Residue mass spectrum in $\mathrm{p}(1 \mathrm{GeV})+{ }^{238} \mathrm{U}$ reactions. Comparison between the predictions of INCL4.5 coupled to ABLA07 (full curve) and experimental data [11-14].

Finally, the excitation functions of residue production cross sections in the incident energy range stretching from $20 \mathrm{MeV}$ to $3 \mathrm{GeV}$, measured in Refs. [15-17], are satisfactorily reproduced, with, however, strong deviations, for a few isotopes.

\section{CONCLUSION}

Compared to the previous version, INCL4.5 has improved on three main points: (i) the inclusion of well documented features of the nucleon and pion average potentials, (ii) the implementation of a dynamical coalescence model for the emission of light charged particles (iii) the exercise of special attention for a good modelling of the first collision. The latter proved to be useful for the improvement of the model for reaction cross section and for low incident energy. The second item is a unique feature of INCL4.5 and allowed a step forward in the prediction of the production of composites of high kinetic energy. The predictive power of the new model has been largely improved.

\section{ACKNOWLEDGMENTS}

This work has been done in the frame of the EU IP EUROTRANS project (European Union Contract $\mathrm{N}^{\circ}$ FI6W-CT-2004-516520). We acknowledge the EU financial support.

\section{REFERENCES}

[1] D. Mancusi et al, "Multifragmentation in spallation reactions", contribution to this conference.

[2] J.-P. Meulders et al, eds., "HINDAS Detailed final report", http://www.theo.phys.ulg.ac.be/wiki/index.php/Cugnon Joseph (2005).

[3] A. Boudard, J. Cugnon, S. Leray and C. Volant, Phys. Rev. C66, 044615 (2002)

[4] A. R. Junghans et al, Nucl. Phys. A629, 635 (1998).

[5] J. Cugnon, A. Boudard, S. Leray, and D. Mancusi, "Results with INCL4", Proc. of Int. Topical Meeting on Nuclear Research Applications and Utilization of Accelerators (AccApp09), Vienna, 2010, ISBN 978-92-0-1504104,SM/SR-02 (2010).

[6] R. E. Prael and M. B. Chadwick, Los Alamos National Laboratory report LA-UR-97-1744, 1997

[7] IAEA, "Benchmark of Spallations Reactions", http://wwwnds.iaea.org/spallations/

[8] A. Budzanowski et al., Phys. Rev. C 78, 024603 (2008).

[9] V. Ricciardi et al, "Results obtained with ABLA07”, Proc. of Int. Topical Meeting on Nuclear Research Applications and Utilization of Accelerators (AccApp09), Vienna, 2010, ISBN 978-92-0-150410-4, SM/SR-04 (2010).

[10] T. Enqvist et al., Nucl. Phys. A686, 481 (2001).

[11] J. Taieb et al., Nucl. Phys. A 724, 413 (2003).

[12] M. Bernas et al., Nucl. Phys. A765, 197 (2006).

[13] M. Bernas et al., Nucl. Phys. A 725, 213 (2003).

[14] M. V. Ricciardi et al., Phys. Rev. C 73, 014607(2006).

[15] Y. E. Titarenko et al., Nucl. Instr. and Meth. A562, 801 (2006).

[16] M. Gloris et al., Nucl. Instr. and Meth. A463, 593 (2001).

[17] I. Leya et al., Nucl. Instr. and Meth. B229, 1 (2005). 